\title{
Retroviral-Based BMP-4 In Vivo Gene Transfer Strategy with Intramedullary Viral Delivery Optimizes Transgene Expression in Rat Femur Fractures
}

\author{
Charles H. Rundle ${ }^{1,2}$, Shin-Tai Chen ${ }^{1,2,3}$, Ryan Porte ${ }^{1}$, Jon E. Wergedal ${ }^{1,2,3}$ and \\ K.-H. William Lau ${ }^{1,2,3, *}$
}

${ }^{1}$ Musculoskeletal Disease Center, Jerry L. Pettis Memorial Veterans Administration Medical Center, 11201 Benton Street, Loma Linda, California, 92357 and ${ }^{2}$ Department of Medicine, ${ }^{3}$ Department of Biochemistry, Loma Linda University, Loma Linda, California, 92350, USA

\begin{abstract}
We have developed an intramedullary delivery strategy to administer retroviral vectors expressing a therapeutic gene to promote healing of a closed rat femur fracture. This strategy involves implantation of an indwelling catheter with the stabilizing Kirschner $(\mathrm{K})$-wire during the surgery prior to fracture of the femur by the three-point bending technique. It uses the openings in the bone that were already created for the stabilizing K-wire and the catheter insertion. In this study, transgene expression and callus bone formation induced by intramedullary delivery of MLV-based vectors expressing the bone morphogenetic protein-2/4 (BMP-2/4) hybrid gene or $\beta$-galactosidase ( $\beta$-gal) gene were compared with those produced by percutaneous injections of the same vectors at the periosteum of the fracture site. The percutaneous injections of MLV-BMP-2/4 vector led to massive but asymmetric transgene expression in surrounding tissues within the fracture callus and large amounts of supraperiosteal as well as asymmetric callus bone formation. In contrast, the intramedullary administration produced a robust and symmetric pattern of transgene expression at the fracture site with very minimal transduction at cells of surrounding tissues, resulting in normal subperiosteal bone development around the entire fracture callus without supraperiosteal bone formation. In summary, we have developed an intramedullary retroviral vector delivery strategy with a rat femur fracture model that led to uniform transgene expression around the entire fracture site, which optimizes the gene therapy-enhanced fracture repair. This strategy should readily be adapted to administer large dosages of any therapeutic vehicle (therapeutic molecules, peptides, or proteins, as well as viral or non-viral vectors) throughout much of early fracture repair, and thus it would be an ideal rat model for in vivo testing of various therapeutic agents to promote fracture repair.
\end{abstract}

\section{INTRODUCTION}

Small animal species have become a preferred model system for the study of molecular mechanisms of bone repair for a number of reasons, including but not limited to, costs, ease of handling, time (small animals heal fractures faster than larger animals) and the availability of essential immunological and molecular reagents. The rodent, and most notably the rat, has traditionally been the model of choice for pharmacological investigations. With respect to bone repair, the closed rat femur fracture described by Bonnerans and Einhorn [1] has become a standard model for studies of the histology and molecular biology of endochondral bone healing. Even with the advent of fracture studies in genetically defined models, such as knockout and transgenic mice, the rat model continues to provide valuable information on endogenous gene expression during fracture repair [2-5] that can be adapted for therapeutic investigations.

While the rat femur fracture model has become essential for observations of the normal course of fracture healing, it

\footnotetext{
*Address correspondence to this author at the Musculoskeletal Disease Center (151), Jerry L. Pettis Memorial VA Medical Center, 11201 Benton Street, Loma Linda, CA 92357, USA; Tel: (909) 825-7084 x 2836; Fax: (909) 796-1680; E-mail: William.Lau@med.va.gov
}

has remained difficult to study the effects of therapeutic intervention. Assessing the efficacy of gene or protein therapy in the rat femur fracture model requires direct injections of therapeutic agents to the fracture site, which have proven to be difficult. Specifically, fracture healing is mediated largely by the periosteum, a tissue that is only a few cells in thickness in early fracture repair [6], and therefore presents a difficult target for therapeutic application during the most clinically relevant time for intervention. Variation in the delivery of therapeutic agents makes evaluation of their efficacy difficult.

The leakage of the anabolic agent or gene vector to the surrounding non-bony tissues during administration is a significant problem if the agent is secreted or capable of promoting transdifferentiation of the non-bony tissues into heterotopic bone, as is the case with the bone morphogenetic proteins (BMPs). This problem was especially evident in our previous in vivo application of BMP-4 gene therapy in the rat femur fracture model; a percutaneous injection of a Moloney leukemia virus (MLV)-based vector expressing a BMP-2/4 hybrid gene produced large amounts of supraperiosteal, heterotopic bone with an asymmetric distribution at the injection site [7]. This result could produce uneven repair and a suboptimal return of bone strength. The percutaneous injec- 
tion approach also limits the amounts of therapeutic agents or vectors that can be administered to the fracture site [8]. Many studies have found it simpler to attempt delivery of larger dosages of therapeutic agent locally to endochondral bone injuries in larger animal subjects [9-11], systemically in the rat femur model [12], or to utilize segmental defect models in the endochondral [13] or membranous bones of rodents $[14,15]$. While some therapeutic approaches might function quite well in a percutaneous delivery, others, such as the secreted and potent osteogenic growth factors secreted from cells transfected with BMP genes, develop ectopic or heterotopic bone without obvious therapeutic benefit $[7,8]$. The evaluation of therapeutic agents for bone healing would benefit from the accurate application of therapeutic agents to the rat femur fracture tissues.

In this report, we describe a novel, relatively simple injection technique for local delivery of a therapeutic gene vector to the standard rat femur fracture model. This approach places a catheter in the intramedullary cavity of the femur during surgery for subsequent administration of the therapeutic retroviral vector to the interior of the fracture site. It utilizes the surgical approaches described in the original procedure and avoids the confounding effects of any injury resulting from additional surgery necessary for the application technique. To demonstrate the utility of this approach, we compared percutaneous and intramedullary applications of a $\beta$-galactosidase ( $\beta$-gal) marker transgene and a BMP-2/4 hybrid transgene [16]. The BMP-2/4 transgene was chosen because 1) of its potent osteogenic activity, which allows efficient and accurate localization of its transduction through the identification of both normal callus and supraperiosteal heterotopic bone, and 2) its effects on fracture studies have been well characterized $[7,8]$. Our results confirmed that this technique confined transgene expression to the subperiosteal tissues and produced a symmetric fracture callus. As such, this approach could be used to evaluate enhanced fracture repair, rather than simply augmented bony tissues in the fracture callus.

\section{MATERIALS AND METHODS}

\section{Animals}

All procedures involving animals were approved by the Institutional Animal Care and Use Committee of the Jerry L. Pettis Memorial VA Medical Center in accordance with the Animal Welfare Act. Fractures were produced in the femurs of male Fischer 344 rats (Harlan, Indianapolis, IN) at 12 weeks of age. Normal healing of the standard closed rat femur fracture model, as defined by bony union of the fracture callus, occurs after 28 days of healing [7]. The initial step of this surgical adaptation is identical to the described rat femur closed fracture method [1], but prior to fracture, a standard 20 gauge (20-G) catheter is inserted into the intramedullary space for postfracture injections.

\section{Prefracture Bone Stabilization and Catheter Insertion}

Surgery was performed under general anesthesia (50 $\mathrm{mg} / \mathrm{kg}$ ketamine, $5 \mathrm{mg} / \mathrm{kg}$ xylazine, delivered intraperitoneally). Two incisions were made at opposite ends of the femur, $0.5-\mathrm{cm}$ in length over the greater trochanter and a 1.0$\mathrm{cm}$ in length lateral to the knee and parallel to the axis of the leg. An additional incision of the muscle tissue separated it from the patellar tendon. The patellar tendon was displaced medially, exposing the condyle, which was then drilled with a Dremel tool using a \#52 drill bit, just sufficient to allow the insertion of the Kirschner (K)-wire. A 1.14-mm diameter Kwire was inserted in retrograde fashion from the condyle through the intramedullary space, penetrating the greater trochanter and exiting through an incision in the overlying skin. The pin was then completely withdrawn and replaced with a 19-G stainless steel \#304 thin wall metal tube [1.07$\mathrm{mm}$ outside diameter $\mathrm{x}$ 0.81-mm inside diameter (Small Parts, Inc., Miami Lakes, FL)] inserted from the condyle and through both the intramedullary space and the greater trochanter. The tip of the catheter needle was inserted into the end of the tube and the tube withdrawn through the condyle while guiding the insertion of the catheter needle and tubing in antegrade fashion into the intramedullary space through the greater trochanter (Fig. 1A). The guide tube was completely removed and replaced with a different $1.14-\mathrm{mm}$ diameter K-wire inserted from the condyle and forced through the original opening in the greater trochanter, squeezing alongside the inserted catheter (Fig. 1B). This K-wire was bent to a short hook and seated around the greater trochanter, pointing away from the catheter needle and tubing so as not to pinch it (Fig. 1C). This approach used the stabilizing $\mathrm{K}$ wire to anchor the catheter so it could not be removed or broken by the animal. The K-wire was cut flush with the condyle at the distal end, the patellar tendon sutured back into place and the skin incisions sutured, but it was not necessary to suture the muscle covering the trochanter. The catheter needle was removed, the catheter hub capped with a Luer Lock cap, and the femur fractured using the routine three-point bending technique (see below). This method placed the catheter in the greater trochanter in a position where the animal could not reach it with its mouth. The wound at the trochanter was treated with antiseptic to minimize the risk of infection.

\section{Femoral Fracture}

A closed midshaft transverse fracture was produced by the three-point bending technique described for the standard femur fracture model by Bonnarens and Einhorn [1]. The entire animal was X-rayed to confirm the success of the stabilization and fracture procedures (Fig. 1D). The animal was observed during recovery until alert and mobile. Analgesia (60 $\mu \mathrm{g} / \mathrm{kg}$ buprenorphine, delivered subcutaneously) was administered. The movement of the animal was not restricted at any time.

\section{MLV-BMP-2/4 or MLV- $\beta$-Gal Control Gene Vector In- jection}

Fig. (2) shows the design of our MLV- $\beta$-gal control and MLV-BMP-2/4 therapeutic vectors. To facilitate extracellular secretion of the mature BMP-4 protein from the transduced cells, a hybrid BMP-2/4 gene was developed in which the native signal sequence of BMP-4 gene was replaced with that of BMP-2 [16,17]. To maximize vector tropism, the vectors were pseudotyped with the vesicular stomatitis virus glycoprotein (VSV-G) envelope. For this study, groups of three animals receiving either control (MLV- $\beta$-gal) or the MLV-BMP-2/4 therapeutic gene vector were compared at weekly intervals throughout fracture healing. 

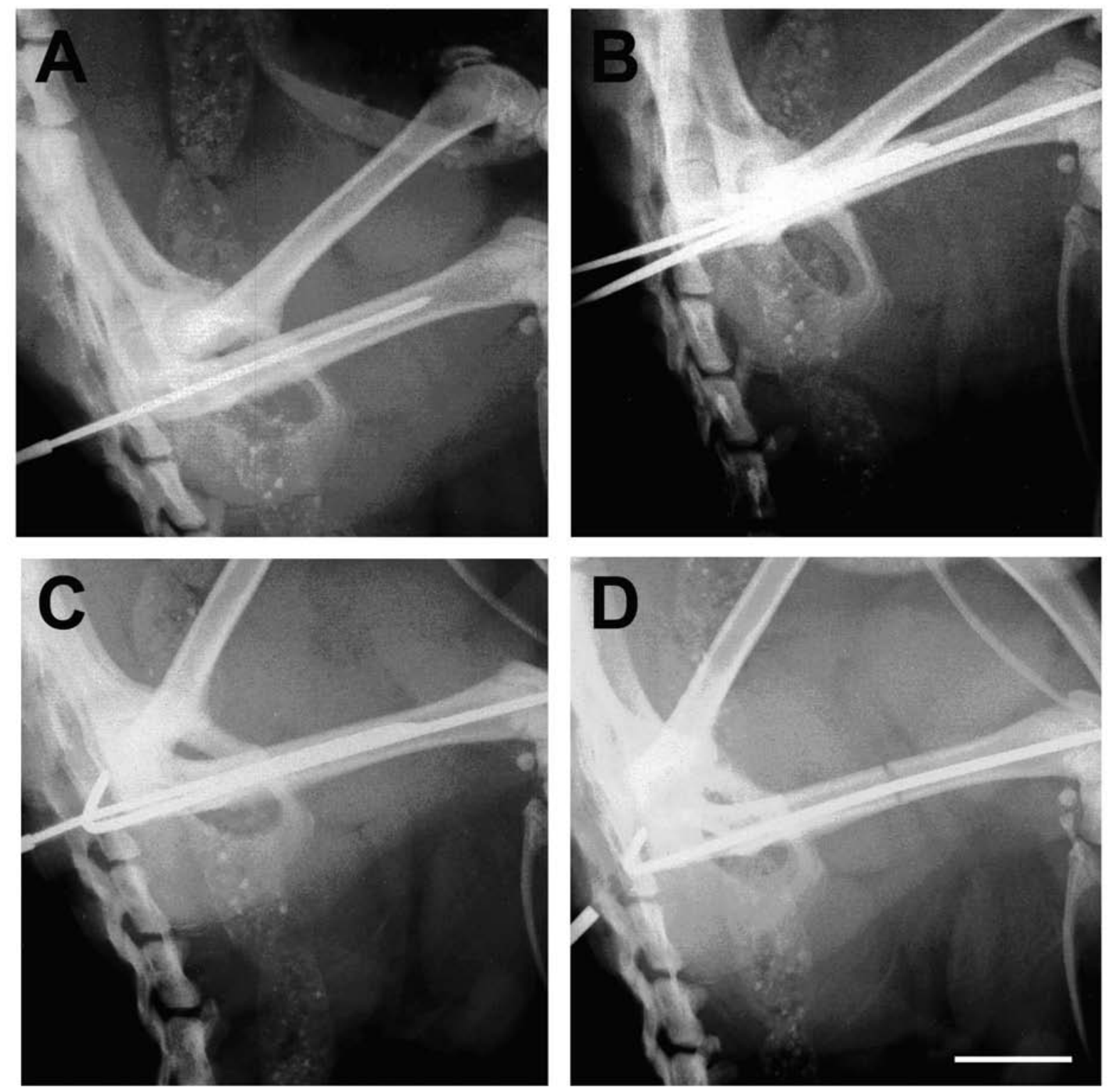

Fig. (1). Modification of the rat femur fracture surgery for intramedullary injection. In both techniques, a stabilizing K-wire is inserted into the medullary space, hooked, seated at the greater trochanter and cut flush with the condyle prior to fracture by three-point bending. In the catheter insertion modification, the pin inserted through the greater trochanter and then immediately removed. A 20-G stainless steel metal tube is inserted in retrograde fashion in its place, and then pulled backward to guide a 20-G catheter and needle into the space from the greater trochanter in the anterograde direction (A). A second K-wire is inserted into the intramedullary space through the condyle and out the greater trochanter, where it squeezes the catheter and needle at the opening in the greater trochanter (B); the entire catheter can be pulled slightly backward to position its end at the midshaft point where the fracture is expected. It is hooked and seated as usual (C). The catheter needle is removed and the fracture produced as usual (D). The fracture and the external catheter tubing and hub are visible, though the internal tubing cannot be visualized by X-ray. Scale bar $=1 \mathrm{~cm}$.

Percutaneous injections of MLV-BMP-2/4 or MLV- $\beta$-gal were performed as previously described [7]. Injection was routinely performed at one day postfracture, when cell proliferation resulting from the injury [18] facilitates MLVbased vector transduction [19]. The rat was administered the therapeutic growth factor gene or reporter gene under general anesthesia. A maximum of $1 \times 10^{9}$ transforming units (tfu) in $150 \mu 1$ total volume was delivered in a single percutaneous injection to the lateral aspect of the fracture site. Two additional animals were injected with a $150 \mu 1$ volume of radio-opaque iodine contrast dye (Conray; Mallinckrodt,
St. Louis, MO) under fluoroscopy to visualize location and distribution of the injected materials (Fig. 3A).

For intramedullary administration, the injection site was treated with an antiseptic, and the catheter plug removed. A 25-G spinal needle was inserted for the injection, and a fluoroscope was again used to insert the needle through the catheter tubing and localize the tip of the needle to just proximal of the fracture site (Fig. 3B). The stylus of the needle was removed, clearing the tip of the needle of any tissue. The fracture was injected with the retroviral vector, intrame- 
A

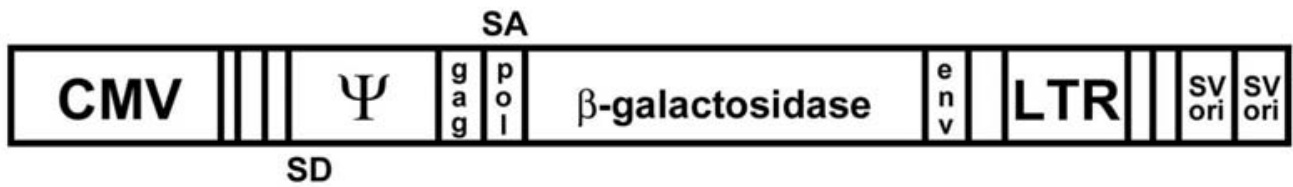

B

SA

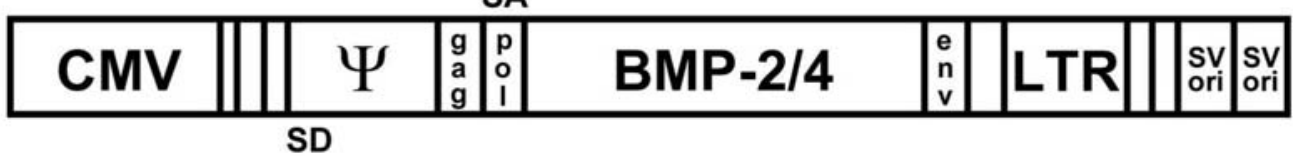

Fig. (2). Schematic structure of the MLV-based vector expressing (A) the marker control $\beta$-gal transgene construct and (B) the BMP-2/4 hybrid therapeutic transgene construct. The CMV early promoter (CMV), which is used to express the genomic RNA of the vector, is not packaged into the vector by the MLV packaging sequence $(\psi)$. SVori contains the sequences of SV40 replication used to increase gene copy numbers during vector production. Upon stable transfection, the 3' long-terminal repeat (LTR) is duplicated 5' of the packaging sequence, from where it drives transgene expression. Most of the sequences of the viral structural protein (gag), envelope protein (env), and retroviral polymerase (pol) genes have been deleted from each gene construct. A splice donor (SD) site is presented in the packaging sequence ( $\psi$ ) and a splice acceptor (SA) site presented in the overlapping sequence of the truncated gag and the truncated pol sequence. Therefore, the large majority of the viral sequences are deleted from the resulting transcript.

dullary and aseptically through the catheter, with a maximum of $1 \times 10^{9}$ tfu of the viral vector (in $150 \mu$ total volume) per animal. Medullary fluid was vented back through the catheter tubing during the injection. At this point, the catheter hub could be cut off or recapped and left in place for additional injections, if necessary, but it was easily removable at any time, if the health of the animal necessitated its removal or if it was no longer needed for injections. It was routinely removed or cut after the injection to minimize the risk of subcutaneous infections, and the wound at this site was treated with antiseptic and resutured, if necessary.

Recovery and analgesia following injection were performed as described for surgery. The animals were observed thereafter for ease of movement or symptoms of discomfort and infection, and the administration of analgesics continued if pain persisted.

\section{Analysis of Transgene Expression and Bone Formation}

Fracture healing was monitored by X-ray analysis in groups of three animals receiving the transgenes at weekly intervals for 28 days postfracture. Animals were briefly immobilized by isoflurane inhalation anesthesia while X-ray images were obtained (Faxitron, Wheeling, IL).

At weekly postfracture times the animals were euthanized by carbon dioxide inhalation. Whole femurs were immersion-fixed in $10 \%$ buffered formalin overnight for analysis of the bone morphology, radiology, localization of $\beta$-gal marker gene expression and an examination of the fracture callus histology. $\beta$-gal expression was determined by standard staining techniques using the substrate X-gal [7] on fracture calluses at one week postfracture, when fracture tissue proliferation permits the degree of transgene expression to be easily visualized. Bones were split open to enhance stain penetration to the fracture tissues.

Fracture callus histology was examined by Masson's Trichrome staining of decalcified and paraffin-embedded sections obtained from the different groups of animals euthanized throughout fracture healing. Trichrome staining was chosen because the staining of the collagen in the demineralized sections provided an accurate representation of the fracture callus bony tissues. Both longitudinal and cross sections were examined.

Cross-sections of Masson's Trichrome stains of the fracture callus histology were quantified for collagen staining at 28 days postfracture by histomorphometry using ImagePro Plus software (Media Cybernetics, Silver Spring, MD). For this evaluation, the callus area excluded all cortical bone and the intramedullary space, but included all other subperiosteal bone, as well as the supraperiosteal bone of the percutaneous injection. Groups of 6 animals that received BMP-2/4 percutaneous transgene injections were compared with groups of 10 and 11 animals that received intramedullary injections of the $\beta$-gal or BMP-2/4 transgenes, respectively. Statistical analysis was performed by two-tailed t-test and results deemed significant at $\mathrm{P}<0.05$.

\section{RESULTS}

\section{Effects of Catheter Implantation on Fracture Production and Animal Health}

The surgical procedure for insertion of the flexible catheter produced a transverse, midshaft fracture that was no different from the standard application of the rat femur fracture (Fig. 1D) [1]. The catheter remained intact; but because it was secured at the trochanter opening by the stabilizing Kwire, it was not removed by the animal, either intentionally or accidentally. The animal appeared to suffer no obvious discomfort or restricted motion, other than that normally observed for the standard femur fracture model. Healing with the catheter in place appeared normal. However, approximately $25 \%$ of the animals receiving the intramedullary injection developed a subcutaneous infection at the site where the catheter tubing penetrated the skin; this infection 
was usually observed by the second week of healing. It usually remained localized, and did not affect healing in an observable way, as the medullary cavities of these animals did not display polymorphonuclear (PMN) cell infiltrations, an indicator of infection. However, more serious infections occasionally progressed to the intramedullary cavity, displaying obviously impaired callus development and accompanying intramedullary PMN infiltrations. Infections could be avoided through prophylactic antibiotic treatments.

\section{Evaluation of Fracture Injection}

A comparison of the percutaneous and intramedullary approaches by injection of a contrast dye revealed that the intramedullary injection volume was delivered internally to the fracture site before being dissipated in the circulation (Fig. 3B, arrow). A percutaneous injection of the same volume of contrast dye revealed that much was dispersed throughout the muscle tissue (Fig. 3A, arrow). A set of three animals each was euthanized at 7 days postfracture to localize the expression of the $\beta$-gal marker transgene after the percutaneous injection (Fig. 3C) or the intramedullary injection (Fig. 3D). The percutaneous injection displayed weak staining of the fracture callus that was limited to a small area of its exterior, i.e., the side of injection (top half bone of Fig. 3C). There was no staining in the interior of the bone (bottom half bone of Fig. 3C), demonstrating the difficulty in targeting subperiosteal layers at the fracture site for percuta- neous injection. In comparison, the intramedullary injection approach displayed a robust staining of both the interior (bottom half bone of Fig. 3D) and the exterior (top half bone of Fig. 3D) of the fracture callus under the same staining conditions. Staining was also symmetric, and encompassed the entire circumference of the fracture. It was, however, restricted to the fracture tissue, indicating that the remainder of the intramedullary canal was not damaged enough by the surgical procedure to allow MLV transduction and expression of the $\beta$-gal marker gene in other areas of the intramedullary space. We have observed occasional staining at the condyle, presumably caused by viral transduction of proliferating cells at the surgical wound and subsequent $\beta$-gal transgene expression. Thus, it is clear that the intramedullary injection strategy produced a strong and symmetric expression of a marker transgene localized to the fracture site, yet successfully propagated into the fracture callus tissues through cell proliferation.

\section{Evaluation of Fracture Therapy}

Fracture healing was also monitored by examination of the X-rays obtained from whole living animals at weekly postfracture intervals (Fig. 4). In both case, the initial bone formation was evident at 7 days postfracture (Fig. 4A,B). The development of supraperiosteal bone was evident by 14 days (Fig. 4C) in the percutaneous injection, and progressively developed through 21 and 28 days (Fig. 4E, arrow in
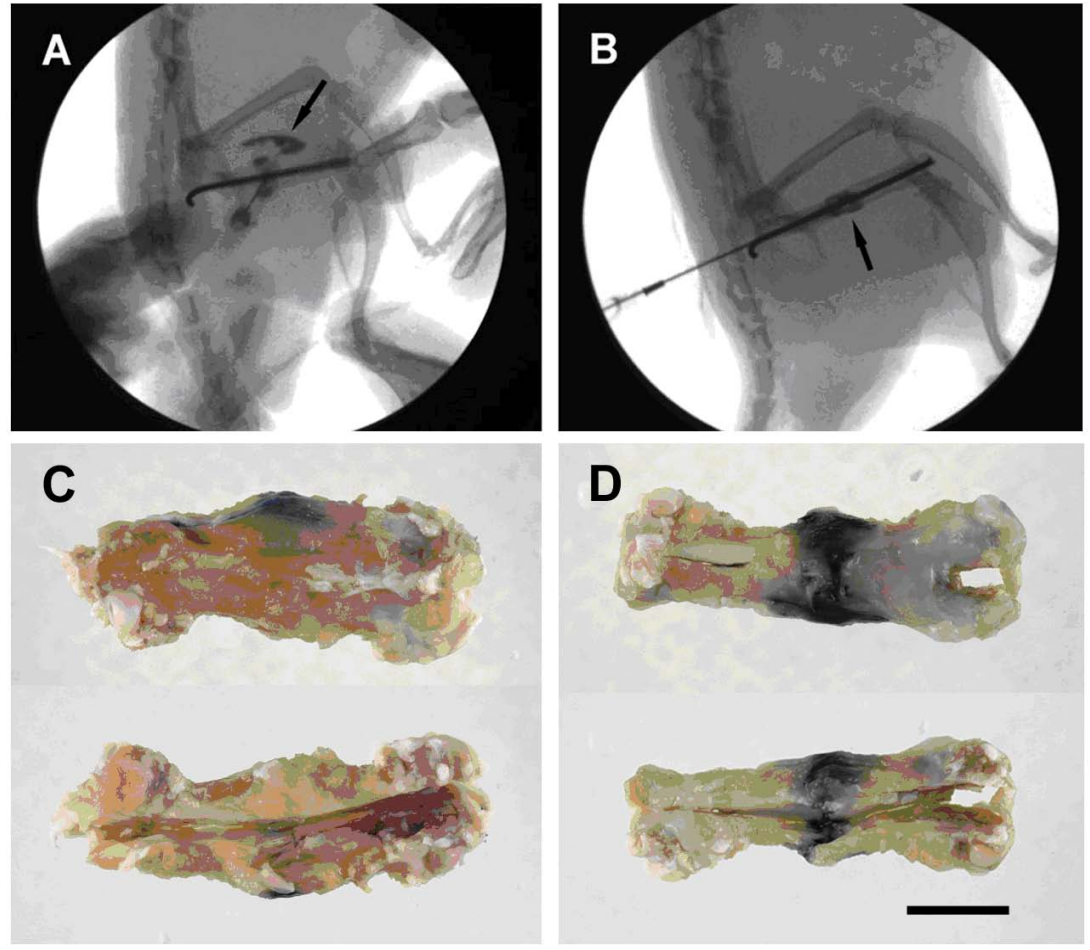

Fig. (3). Marker dye (A,B) or gene $(\mathbf{C , D})$ localization in fractures injected from the percutaneous (lateral) aspect or through the intramedullary (catheter) injection. A and $\mathbf{B}$ : A fluoroscope was used to visualize a radio-opaque contrast dye during a percutaneous injection from the lateral aspect (A) or an intramedullary catheter injection (B). Despite best efforts, the dye immediately distributed in the leg muscles when injected from the exterior (A, arrow). When injected through the catheter, the contrast dye was retained in the medullary space (B) close to the fracture site (arrow). C and D: The MLV-based vector expressing the $\beta$-gal marker transgene was injected at one day postfracture and the femurs harvested at 7 days postfracture, split open and stained for marker expression for both the percutaneous $(\mathbf{C})$ and intramedullary (D) injections. The intramedullary injection (D) produced more extensive and symmetric marker expression in the exterior (D, top) and interior $(\mathbf{D}$, bottom $)$ of the fracture than the percutaneous injection exterior $(\mathbf{C}$, top $)$ or interior $(\mathbf{C}$, bottom). $S$ cale bar $=1 \mathrm{~cm}$. 
Fig. 4G). Small amounts of supraperiosteal bone also developed following the intramedullary injection (Fig. 4D,F, arrow in $\mathbf{H}$ ), but this was presumably due to a slight leakage of the therapy through the wound at the condyle. Such leakage did not seem to affect the the fracture callus and was limited, as $\beta$-gal staining at the wound in the condyle was minimal (Fig. 3D, bottom). Otherwise, the bone resulting from the intramedullary injection of MLV-BMP-2/4 was confined to the fracture callus, where it appeared to be filling the gap over the fracture site as healing progressed (Fig. 4D,F).

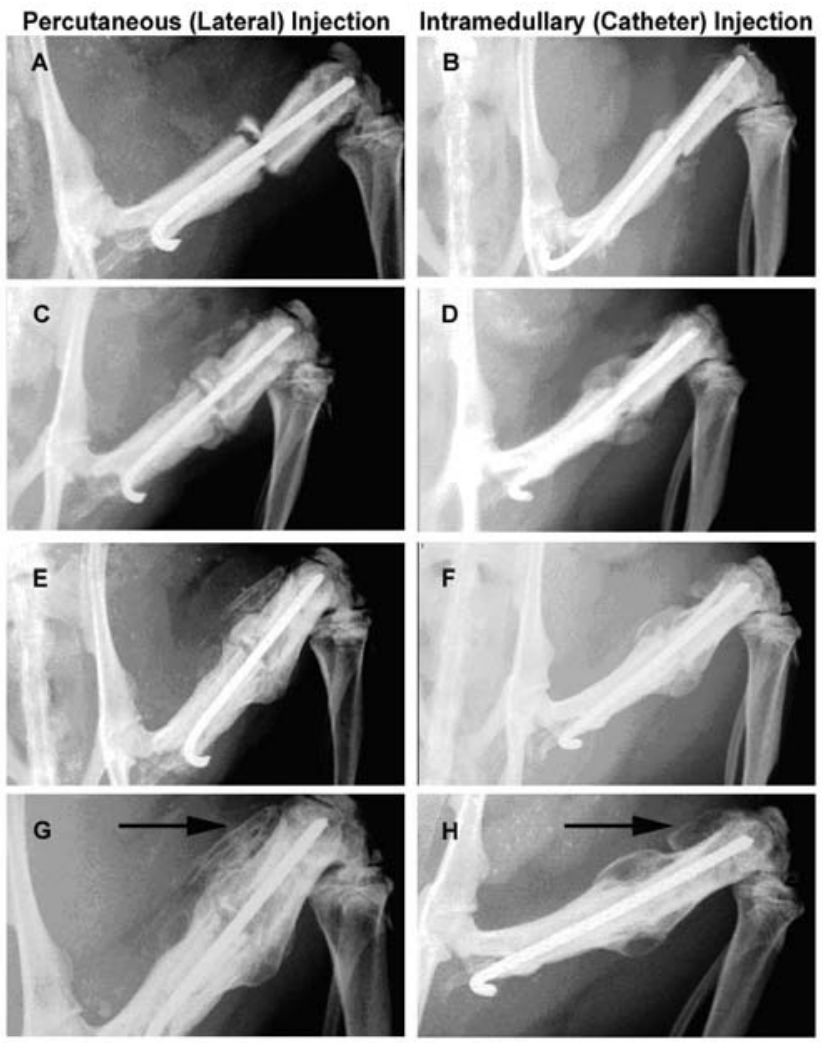

Fig. (4). X-ray comparison of mineralized tissue during healing. The injection was performed from the exterior (percutaneous, left) or through the catheter (intramedullary, right) and monitored at 7 days $(\mathbf{A}, \mathbf{B}), 14$ days $(\mathbf{C}, \mathbf{D}), 21$ days $(\mathbf{E}, \mathbf{F})$ and 28 days $(\mathbf{G}, \mathbf{H})$ healing. These X-rays follow the healing in the same animal for each injection technique. Some supraperiosteal bone has developed from leakage of the therapy at the condyle, most obvious in $\mathbf{H}$ (arrow), but it is not nearly as extensive as that of the percutaneous injection in $\mathbf{G}$ (arrow).

An examination of the fractured femurs obtained from animals after 14 days and 28 days of healing also suggested that both injection approaches produced normal bone, but the percutaneous injection also produced heterotopic supraperiosteal bone (radiology and histology in Fig. 5A,C), while the intramedullary injection produced normal subperiosteal bone at the fracture site (radiology and histology in Fig. 5B,D). Large portions of the lateral aspect of the femur appeared ossified when the MLV-BMP-2/4 therapy was applied percutaneously. While both approaches produced callus bone that eventually resolved to normal, the intramedullary injection confined the bone to the subperiosteal layers of the fracture callus, where the newly formed bone was incorporated into the developing fracture callus.
Examination of fracture callus cross-sections suggested that the additional bone was incorporated into the subperiosteal layers of the developing fracture callus when therapy was applied to the intramedullary space (Fig. 6A). When quantified by histomorphometry at 28 days postfracture, a time just before bony union occurs in this fracture model, the cross-sectional callus area was determined to be significantly greater in the percutaneous injection, although it included supraperiosteal bone (Fig. 6B). However, the area of bone per area of callus, which excluded the bone of the cortical and intramedullary areas, was significantly greater in the intramedullary injections (Fig. 6C). The intramedullary approach therefore produced more bone within the periosteal borders of the fracture callus.

\section{DISCUSSION}

We had previously developed a technically simple and relatively non-invasive MLV-based in vivo gene therapy strategy involving a single percutaneous injection of a small amount of MLV-based vector into the periosteum to promote fracture healing in the standard rat femur fracture model [7,20]. Our initial application of this MLV-based in vivo gene therapy with a modified BMP-4 (BMP-2/4) gene yielded an enormous augmentation of histologically normal callus bone formation that eventually remodeled to normal cortical bone. While effective for promoting callus bone formation, it had two potential pitfalls [7]. First, the considerable cortical width and density of the rat femur presented a very difficult target to penetrate with a needle, and it was quite difficult to accurately administer therapy by percutaneous injections, even with the benefit of fluoroscopy. This problem was compounded by the unique ability of BMPs to transdifferentiate muscle cells into bone cells. Accordingly, "mis-injection" of the MLV-BMP-2/4 retroviral vector caused massive supraperiosteal heterotopic bone formation at the injection site [7] that did not enhance the bone strength because of its abnormal location. The second pitfall related to the observation that single injections of the retroviral vector to the fracture always resulted in asymmetric viral transduction of target cells and asymmetric transgene expression that was restricted to the injection site [7]. The resulting bone formation was also asymmetrical and restricted to the injected aspect of the fracture (Fig. 3A and Fig. 5A,C). Injections at multiple sites were more technically challenging and did not significantly improve these conditions (data not shown). The use of an alternative osteogenic growth factor gene that did not induce transdifferentiation of muscle cells into bone, such as cyclooxygenase-2 [14] fibroblast growth factor-2 [21], or transforming growth factor- $\beta$ [22], reduced the supraperiosteal heterotopic bone formation but not the asymmetric transgene expression. Thus, we concluded that an improvement in the retroviral vector delivery was necessary to yield more uniform viral transduction and improve the overall utility of our MLV-based in vivo gene therapy strategy for fracture repair studies.

In this study, we explored an intramedullary injection strategy to deliver retroviral vectors to the fracture site. The surgical adaptation of the standard rat femur fracture model as described in the Materials and Methods section allowed the implantation of a medical catheter to guide an intramedullary injection of the retroviral vector to the fracture through the marrow space of the femur. The intramedullary 


\section{Percutaneous Injection}
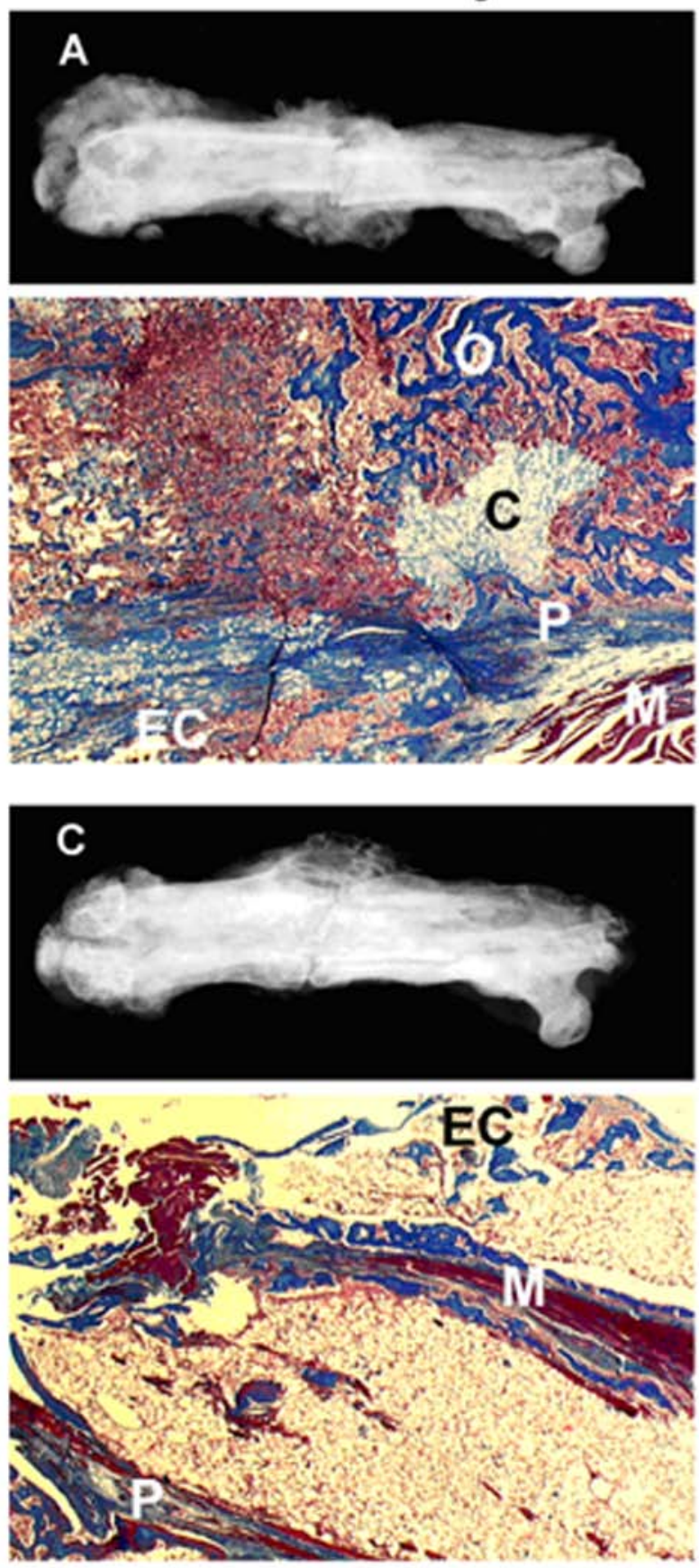

\section{Intramedullary Injection}
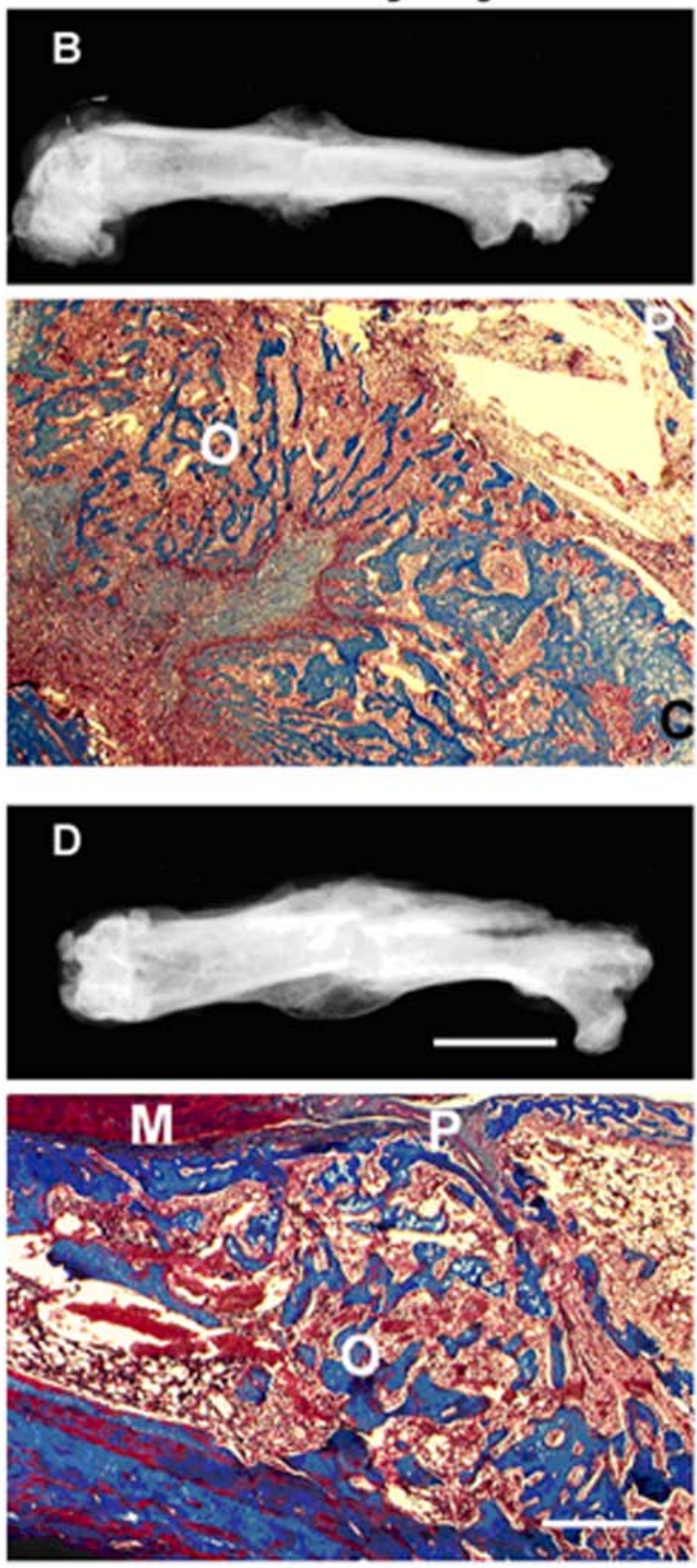

Fig. (5). X-ray and histology of the fracture callus following percutaneous injection from the lateral aspect (left) or intramedullary injection (right) at 14 days $(\mathbf{A}, \mathbf{B})$ and 28 days $(\mathbf{C}, \mathbf{D})$ healing. Scale bar $=1 \mathrm{~cm}$. Trichrome stains of the fracture callus revealed heterotopic (EC) bone formation outside the periosteum $(\mathrm{P})$ at 14 and 28 days in postfracture lateral injections. Bone formation was inferior to the periosteum (P), at 14 and 28 days in postfracture intramedullary injections and did not involve the muscle (M). The fracture tissues appeared normal at all healing times. Cartilage (C), Osteoid (O). Scale bar $=100$ um.

catheter was positioned to deliver the injected viral vector to areas close to the fracture gap. This strategy utilized the wounds already present from the insertion of the K-wire insertion for fracture stabilization to insert the catheter and avoided producing additional damage at the fracture site.

Our rationale for this approach was two-fold: First, previous attempts at injections of intramedullary ex vivo therapy in the mouse [23] revealed a very strong tendency for the high intrinsic intramedullary pressure to push the cell injections back out of the marrow space. Accordingly, we concluded that in a fractured bone the intramedullary pressure would force the injection out of the marrow space through the fracture gap to infect the proliferating periosteal cells, especially when delivered close to the fracture site (Fig. 3B,D). Thus, this process would yield a more uniform viral 
infection of fracture tissues than percutaneous injections. Our findings that intramedullary application of the MLV- $\beta$ gal marker vector to the rat femur fracture by our strategy indeed led to robust and symmetric expression of the $\beta$-gal gene around the entire fracture site (Fig. 3C,D) are entirely consistent with this premise. The fact that the MLV-mediated $\beta$-gal gene expression was confined to the immediate area of the fracture was also consistent with enhanced cell proliferation at the fracture site during the early fracture healing $[18,19]$. Although we occasionally observed staining for $\beta$-gal gene expression at the condyle, where a wound created by K-wire and catheter insertions presumably allowed sufficient proliferation for MLV transduction and transgene expression, such staining was very minimal (Fig. 3). The second rationale was based on our belief that it would be much easier and more consistent to administer therapeutic agents through an anchored intramedullary catheter than by a single percutaneous injection. The intramedullary injection strategy would also allow administration of repeated and much larger volumes of the therapeutic agent than the percutaneous injection approach. This would allow the flexibility to study a wider dose range of the therapeutic agent, a significant investigative advantage over the single percutaneous injection approach.

As an initial assessment of injection strategy, we compared the endochondral bone formation response induced by the intramedullary injection strategy with that promoted by a single percutaneous injection. We confirmed that a single percutaneous injection of the MLV-BMP-2/4 vector to the fracture periosteum induced massive supraperiosteal bone formation by 14 and 28 days of healing (Fig. 5A and $\mathbf{C}$ ), and that the induced callus bone formation was asymmetric and restricted to the injected aspect of the fracture callus. However, the intramedullary MLV-BMP-2/4 vector injection promoted only subperiosteal callus formation with no discernable amounts of supraperiosteal bone formation (Fig. 5B,D). Consistent with the occasional staining for $\beta$-gal gene expression at the condylar wound of the MLV- $\beta$-gal-injected fracture (Fig. 3D), we occasionally observed some heterotopic bone at this area of the MLV-BMP-2/4-treated fractures (Fig. 4D,F,H). Nevertheless, such observations were infrequent and did not affect the callus development; the great bulk of the therapy was confined to the subperiosteal fracture tissue. More excitingly, we noted that callus bone developed in a symmetric pattern along the entire fracture in the fracture calluses injected through the intramedullary catheter, while it developed in a patchy and largely supraperiosteal pattern localized primarily at and around the injection site when injected percutaneously. These findings clearly indicated that our novel intramedullary application strategy produced uniform transgene expression and enhanced callus bone formation in the rat femur fracture model, but avoided supraperiosteal bone formation. In both types of injections, the $\beta$-gal control gene produced fracture healing characteristic of normal healing without therapeutic intervention. These findings support our contention that the $\mathrm{K}$-wire and/or the catheter did not significantly impede the normal fracture healing process. Though we have not pursued the analysis of the callus bone from the intramedullary BMP-2/4 gene therapy further, healing appeared to be progressing to a normal bony union when the therapy was injected through this route.
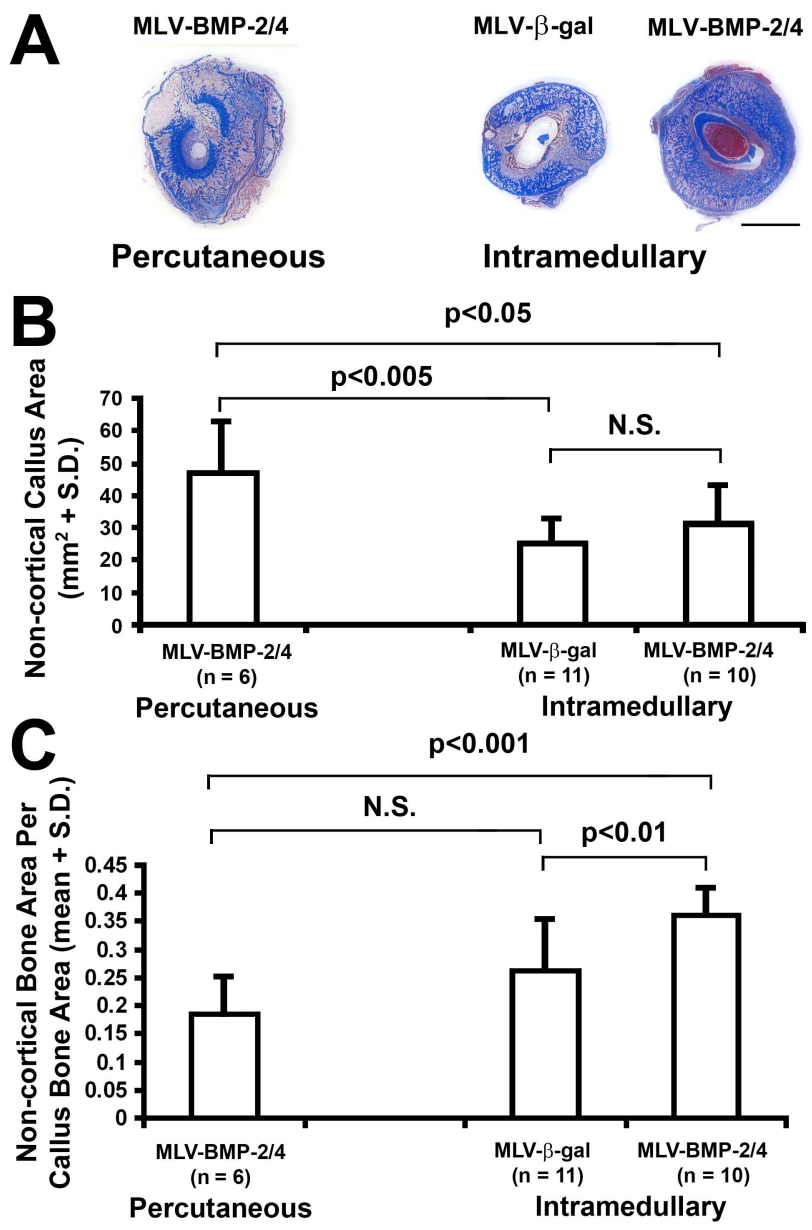

Fig. (6). Cross-sectional histology of the fracture callus (A) following lateral injection (left) or intramedullary injection (right) 28 days healing. The cross-sectional callus area (B) and the cross-sectional area of bone per callus area $(\mathbf{C})$ were quantified by histomorphometry that excluded the cortical bone and intramedullary space from the analysis. Scale bar $=3.0 \mathrm{~mm}$.

Because our original objective of this study did not include the testing of mechanical strength of the healing calluses as an endpoint, this study did not allow a definitive determination as to whether the intramedullary administration strategy improved the mechanical strength of the healing calluses relative to the percutaneous injection approach. Consequently, while our study has provided evidence that the intramedullary injection strategy optimizes transgene expression and yielded uniform subperiosteal callus bone along the entire fracture gap, it remains unclear whether these effects were indeed translated into improved bone strength in the healing fractures. This very important issue, which is relevant to the potential clinical relevance of this strategy, will be investigated in future studies.

One of the concerns of the intramedullary administration strategy is the significant infection hazard presented by an indwelling catheter. Indeed, we have found that infections did occur in some of the animals. However, because of the relatively short duration of this study, most infections remained confined to the subcutaneous layers. We routinely cut the catheter tubing immediately after a single intramedullary injection and closed the skin to minimize infections. 
Should longer postfracture observation times or multiple injections of therapeutic agents require that the catheter be retained, infection can be controlled by the prophylactic administration of antibiotics. The animals displayed no other discomfort related to the catheter implant, and if not removed after injection, the hub routinely remained attached for more than two weeks after surgery. While this study focused on the administration of MLV-based retroviral vectors to the fracture calluses through the intramedullary injection strategy, this strategy could be easily adapted for the administration of other therapeutic vehicles, including therapeutic proteins, peptides, and/or small molecules, as well as other viral vectors or non-viral vectors. However, with respect to other animal fracture models, we should note that our adaptation of rat fracture therapy delivery is not applicable to the mouse femur because of its smaller size and increased fragility.

In summary, we have adapted the originally described standard rat femur fracture technique to develop a novel intramedullary injection strategy for delivering therapeutic vehicles to the fracture site through a surgically implanted catheter. We have clearly demonstrated that this intamedullary injection strategy was superior to our previous percutaneous injection approach in producing optimized transgene expression throughout the entire fracture site without supraperiosteal heterotropic bone formation. This method should be useful in our continuing efforts to develop an effective modality for fracture repair and for improved investigations of the various molecular aspects of the growth factor-induced fracture repair.

\section{ACKNOWLEDGMENTS}

This work was supported in part by a special appropriation to the Jerry L. Pettis Memorial VA Medical Center Musculoskeletal Disease Center. All work was performed in facilities provided by the Department of Veterans Affairs. The authors acknowledge the assistance with surgery provided by Yuji Kasukawa, Xaoguang Wang and Jann Smallwood, and the assistance with histology provided by Nancy Lowen.

\section{REFERENCES}

Bonnarens F, Einhorn TA. Production of a standard closed fracture in laboratory animal bone. J Orthop Res 1984; 2: 97-101.

[2] Meyer RA Jr, Meyer MH, Tenholder M, Wondracek S, Wasserman $\mathrm{R}$, Garges P. Gene expression in older rats with delayed union of femoral fractures. J Bone Joint Surg Am 2003; 85: 1243-54.

[3] Nakazawa T, Nakajima A, Seki N, et al. Gene expression of periostin in the early stage of fracture healing detected by cDNA microarray analysis. J Orthop Res 2004; 22: 520-5.

[4] Li X, Quigg RJ, Zhou J, Ryaby JT, Wang H. Early signals for fracture healing. J Cell Biochem 2005; 95: 189-205.
[5] Rundle $\mathrm{CH}$, Wang $\mathrm{H}, \mathrm{Yu} \mathrm{H}$, et al. Microarray analysis of gene expression during the inflammation and endochondral bone formation stages of rat femur fracture repair. Bone 2006; 38: 521-9.

[6] Bolander ME. Regulation of fracture repair by growth factors. Proc Soc Exp Biol Med 1992; 200: 165-70.

[7] Rundle CH, Miyakoshi N, Kasukawa Y, et al. In vivo bone formation in fracture repair by direct retroviral-based gene therapy with BMP-4. Bone 2003; 32: 591-601.

[8] Einhorn TA, Majeska RJ, Mohaideen A, et al. A single percutaneous injection of recombinant human bone morphogenetic protein-2 accelerates fracture repair. J Bone Joint Surg Am 2003; 85: 142535 .

[9] Keller J, Klamer A, Bak B, Suder P. Effect of local prostaglandin E2 on the fracture callus in rabbits. Acta Orthop Scand 1993; 64: 59-63.

[10] Baltzer AW, Lattermann C, Whalen JD, et al. Potential role of direct adenoviral gene transfer in enhancing fracture repair. Clin Orthop Relat Res 2000; 379 Suppl: S120-5.

[11] Kawaguchi H, Nakamura K, Tabata Y, et al. Acceleration of fracture healing in nonhuman primates by fibroblast growth factor- 2 . J Clin Endocrinol Metabol 2001; 86: 875-80.

[12] Alkhiary YM, Gerstenfeld LC, Krall E, et al. Enhancement of experimental fracture-healing by systemic administration of recombinant human parathyroid hormone (PTH 1-34). J Bone Joint Surg Am 2005; 87: 731-41.

[13] Oest ME, Dupont KM, Kong HJ, Mooney DJ, Guldberg RE. Quantitative assessment of scaffold and growth factor-mediated repair of critically sized bone defects. J Orthop Res 2007; 25: 941-50.

[14] Gysin R, Wergedal JE, Sheng MH-C, et al. Ex vivo gene therapy with stromal cells transduced with a retroviral vector containing the BMP4 gene completely heals critical size calvarial defect in rats. Gene Ther 2002; 9: 991-9.

[15] Spector JA, Luchs JS, Mehrara BJ, Greenwald JA, Smith LP, Longaker MT. Expression of bone morphogenetic proteins during membranous bone healing. Plast Reconstr Surg 2001; 107: 124-34.

[16] Peng H, Chen S-T, Wergedal JE, et al. Development of an MFGbased retroviral vector system for secretion of high levels of functionally active human BMP4. Mol Ther 2001; 4: 95-104.

[17] Chen S-T, Gysin R, Kapur S, Baylink DJ, Lau K-HW. Modifications of the fibroblast growth factor-2 gene led to a marked enhancement in secretion and stability of the recombinant fibroblast growth-2 protein. J Cell Biochem 2007; 100: 1493-508.

[18] Iwaki A, Jinguishi S, Oda Y, et al. Localization and quantification of proliferating cells during rat fracture repair: Detection of proliferating cellular nuclear antigen by immunohistochemistry. J Bone Miner Res 1997; 12: 96-102.

[19] Roe TY, Reynolds TC, Yu G, Brown PO. Integration of murine leukemia virus DNA depends on mitosis. EMBO J 1993; 12: 2099108.

[20] Rundle CH, Strong DD, Chen S-T, et al. Retroviral-based gene therapy with cyclooxygenase-2 promotes the union of bony callus tissues and accelerates fracture healing in the rat. J Gene Med 2008; 10: 229-41.

[21] Jingushi S, Heydemann A, Kana SK, Macey LR, Bolander ME. Acidic fibroblast growth factor (aFGF) injection stimulates cartilage enlargement and inhibits cartilage gene expression in rat fracture healing. J Orthop Res 1990; 8: 364-71.

[22] Joyce ME, Roberts AB, Sporn MB, Bolander ME. Transforming growth factor- $\beta$ and the initiation of chondrogenesis and osteogenesis in the rat femur. J Cell Biol 1990; 110: 2195-207.

[23] Zhang XS, Linkhart TA, Chen S-T, et al. Local ex vivo gene therapy with bone marrow stromal cells expressing human BMP4 promotes endosteal bone formation in mice. J Gene Med 2004; 6: 415 . 